\title{
Avaliação da ação da Aroeira (Schinus terebinthifolius Raddi) na cicatrização de feridas cirúrgicas em bexiga de ratos
}

\author{
Evaluation of the aroreira (Schinus terebinthifolius Raddi) in the healing process of surgical \\ incision in the bladder of rats
}

\begin{abstract}
Periguari Luiz Holanda de Lucena ${ }^{3}$, Jurandir Marcondes Ribas Filho ${ }^{4}$, Marcelo Mazza ${ }^{4}$, Nicolau Gregori Czeczko , Ulrich Andreas Dietz ${ }^{4}$, Mario Adolfo Correa Neto ${ }^{5}$, Gilberto Simeone Henriques ${ }^{4}$, Orlando José dos Santos ${ }^{2}$, Álvaro Pigatto Ceschin ${ }^{6}$, Edilson Schwansee Thiele ${ }^{6}$
\end{abstract}

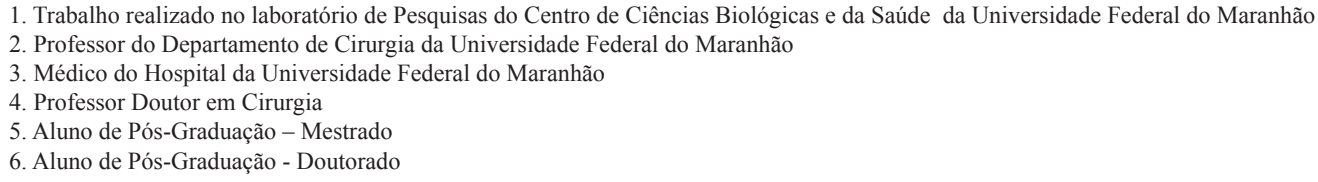

\section{RESUMO}

Objetivo: Avaliar a ação cicatrizante do extrato hidroalcoólico da Aroeira em bexigas de ratos. Métodos: Foram utilizados 40 ratos machos da linhagem Wistar, divididos em dois grupos de 20, denominados grupo Aroeira (GA) e grupo controle (GC). Todos foram submetidos à incisão abdominal mediana com cistotomia de $1 \mathrm{~cm}$, seguida de cistorrafia em plano único com pontos separados de poliglactina 910 5-0 $\left(\right.$ Vicryl $\left.^{\circledR}\right)$. Após este procedimento, nos animais GA injetou-se 100mg por quilo de peso de extrato hidroalcoólico da Aroeira na cavidade peritoneal e nos GC injetou-se $1 \mathrm{ml}$ por quilo de peso de solução salina a $0,9 \%$. Cada grupo foi dividido em dois sub-grupos de 10 animais de acordo com a data da morte: sub-grupo A3 e C3, sacrificados no $3^{\circ}$ dia pós-operatório e sub-grupo A7 e C7, sacrificados no $7^{\circ}$ dia. A parede, a cavidade abdominal e a sutura da bexiga foram avaliadas macroscopicamente. Amostras de tecido da bexiga foram retiradas e analisadas histologicamente, utilizando a coloração de Hematoxilina-Eosina (HE) e tricrômio de Masson. Resultados: Na análise macroscópica observou-se infecção na incisão cirúrgica em três ratos do GC e em um do GA, e aderências peritoneais em 29 ratos do GC controle e 17 no GA. A avaliação microscópica mostrou processo inflamatório agudo mais severo no $3^{\circ}(p=0,045)$ e no $7^{\circ}$ dia $(p=0,002)$. Evidenciou-se ainda diferença estatística nos parâmetros utilizados para a avaliação histológica da cicatrização da bexiga nas variáveis colagenização $(p=0.001)$, reepitelização $(p=0,046)$ e neoformação ( $p$ $=0$ ) nos subgrupos GC e GA no $3^{\circ}$ dia e na variável neoformação vascular ( $\left.p=0,001\right)$ no subgrupo do $7^{\circ}$ dia. Conclusão: O uso de extrato hidroalcoólico de Aroeira mostrou efeito cicatrizante favorável nas cistotomias em ratos.

Descritores: Schinus terebinthifolius. Cicatrização de Feridas. Bexiga. Ratos

\begin{abstract}
Purpose: To evaluate the hydroalcoholic Aroeira extract in the healing process of surgical incisions in the bladder of rats in an experimental comparative study. Methods: Forty male Wistar rats were used. All of them underwent a median abdominal incision, with an $1 \mathrm{~cm}$ cistotomy, followed by one plan suture with separated stitches of poliglactine 910 5-0. The animals were then divided into two groups of 20 rats each, and named the Aroeira group (GA) and the control group (GC). In the first one, $80 \%$ hydro-alcoholic plant extract was injected in the peritoneal cavity, in a one dose of $100 \mathrm{mg} / \mathrm{kg}$. The second group, a $0.9 \%$ saline solution was injected in place of Aroeira extract. Each group was divided into two subgroups (SGA and SGC) of 10 animals. According to their scheduled death, they were named subgroups SGA3 and SGC3 when killed in the 3th day after surgery and subgroups SGA7 and SGC7 in the 7th day. The abdominal cavity and the bladder suture were macroscopically evaluated. Results: The microscopic analysis of the surgical incision in the bladder was performed by means of the hematoxilin-eosine stain and the trichrome of Masson. The macroscopic analysis showed an infection in the surgical incision in three rats of the SGC group and in one of the SGA, and peritoneal adhesion in the 26 rats belonging nine to SGC and 17 in the SGA. The microscopic evaluation revealed a more severe acute inflammation process in the SGC on the 3 th $(\mathrm{p}=0.045)$ and in the 7 th $(0=0.002)$ days. In the SGA, a more significant colagenization $(\mathrm{p}=0.001)$ could be seen, as far as the evidence of vascular neoformation $(p=0.002)$ on the 3 th day. Chronic inflammatory reaction $(p=0.006)$ and a more intense vascular neoformation $(\mathrm{p}=0.001)$ were observed in the 7 th day. Conclusion: The hydroalcoholic Aroeira extract have a favorable effect in the healing process of cystotomies done in rats.
\end{abstract}

Key words: Schinus terebinthifolius. Wound Healing. Bladder. Rats 


\section{Introdução}

O hábito de utilização das plantas medicinais é prática comumente observada em países em desenvolvimento e vem se propagando nos desenvolvidos, nos quais a medicina alopática é a mais utilizada ${ }^{1}$.

O crescente interesse da comunidade científica pela fitoterapia nas duas últimas décadas, levou ao desenvolvimento de várias pesquisas baseadas em práticas populares $^{2}$. A Organização Mundial de Saúde estima que 80\% da população do planeta, de algum modo, utiliza plantas medicinais como medicamentos. Estima-se, também, que 25 mil espécies de plantas sejam usadas nas preparações da medicina tradicional ${ }^{3}$.

O uso medicinal da Aroeira (Schinus terebinthifolius Raddi) é descrito há muitos anos e referido desde a primeira edição da Pharmacopéia Brasileira (1926). Ele é da família Anacardiaceae sendo comum da vegetação litorânea do nordeste brasileiro deste o Rio Grande do Norte até Sergipe ${ }^{4,5}$. Diversas pesquisas têm evidenciado as propriedades antiinflamatórias, antimicrobianas e cicatrizante desta planta ${ }^{6,7,8}$.

Em função do número crescente de profissionais e pacientes utilizando plantas medicinais - mais especificamente a Aroeira - para o tratamento de várias doenças e ainda pelo pequeno número de trabalhos científicos existentes na literatura respaldando esta terapêutica, realizou-se este trabalho que tem como objetivo avaliar a ação cicatrizante do extrato hidroalcoólico da Aroeira em bexiga de ratos através de análise macroscópica e histológica.

\section{Métodos}

Este trabalho foi realizado no Laboratório de Pesquisa do Departamento de Fisiologia e Farmacologia do Centro de Ciências Biológicas e da Saúde da Universidade Federal do Maranhão (UFMA) e foi aprovado pelo Comitê de Ética em Pesquisa da UFMA.

\section{Preparo do extrato hidroalcoólico da Aroeira}

Utilizou-se a Aroeira (Schinus terebinthifolius Raddi) provenientes da Fazenda Santa Ana em Palmeirantes (Tocantins). A parte da planta utilizada foi a entrecasca submetida a moagem, obtendo-se um pó, o qual foi diluído com álcool absoluto e água destilada formando $3450 \mathrm{ml}$ de extrato bruto. Este extrato foi concentrado em vapor rotativo sob pressão reduzida a temperatura de 55 a $60^{\circ}$ para eliminação total do solvente. $\mathrm{O}$ material obtido depois da concentração apresentou-se em forma de pasta. Dela foram retirado $2,8 \mathrm{~g}$ do extrato bruto etanólico ou hidroalcoólico, a seguir diluido em $14 \mathrm{ml}$ de solução salina resultando em concentração de $100 \mathrm{mg} / \mathrm{ml}$. Ele foi armazenado em refrigerador a $10^{\circ} \mathrm{C}, \mathrm{com}$ validade de 1 ano para uso.

\section{O experimento}

Foram utilizados 40 ratos (Rattus norvegicus albinus) da linhagem Wistar, machos com peso variando entre 123 e 308 gramas. Eles foram divididos aleatoriamente em dois grupos de 20 denominados grupo controle (GC) e grupo Aroeira (GA) que por sua vez foram divididos em sub-grupos de 10 animais (SGC e SGA) e de acordo com as datas da morte, estabelecida no $3^{\circ}$ (SGC3 ou SGA3) e $7^{\circ}$ (SGC7 ou SGA7) dias do pós-operatório.

Para a anestesia, o animal foi colocado em uma campânula plástica contendo algodão umedecido em éter sulfúrico, mantido em sistema fechado. Quando o rato assumia a posição deitada e ficava imóvel era retirado e pesado. A seguir era posicionado em decúbito dorsal sobre um suporte de madeira e fixado com fitas adesivas. A manutenção da anestesia para o procedimento era feita em sistema semifechado com algodão embebido em éter.

Com o animal posicionado, realizou-se tricotomia na região do terço inferior do abdômen seguida de antissepsia com Polivinilpilorridona-iodo (Povidine ${ }^{\circledR}$ ) e colocação de campo cirúrgico. Foi realizada incisão mediana suprapúbica de $2 \mathrm{~cm}$ com bisturi lamina $\mathrm{n}^{\circ} 15 \mathrm{em}$ pele e tecido celular subcutâneo; os planos musculoaponeurótico e o peritônio foram incisados com tesoura. Aberta a cavidade abdominal, a bexiga foi identificada, exposta (Figura 1) e submetida a uma cistostomia de $1 \mathrm{~cm}$ na cúpula vesical seguida de cistorrafia em plano único extramucoso, com quatro pontos separados e quatro seminós utilizando-se fio 5-0 de poliglactina $910\left(\right.$ Vicryl $\left.^{\circledR}\right)$. Nos animais do GC, após a cistorrafia, injetou-se na cavidade peritoneal, em dose única, solução salina a $0,9 \%$ na proporção de $1 \mathrm{ml}$ por quilo de peso. No GA, injetou-se o extrato hidroalcoólico da Aroeira na concentração de $100 \mathrm{mg} / \mathrm{ml}$ sendo a dose calculada em 100 mg por quilo de peso.

A síntese da parede abdominal foi realizada com náilon 4-0 com agulha cilíndrica de 1,5 cm $\left(\right.$ Mononylon $\left.^{\circledR}\right)$. Utilizou-se sutura contínua para o plano músculo-aponeurótico e pele separadamente. Após o procedimento, a ferida foi limpa com Povidine ${ }^{\circledR}$.

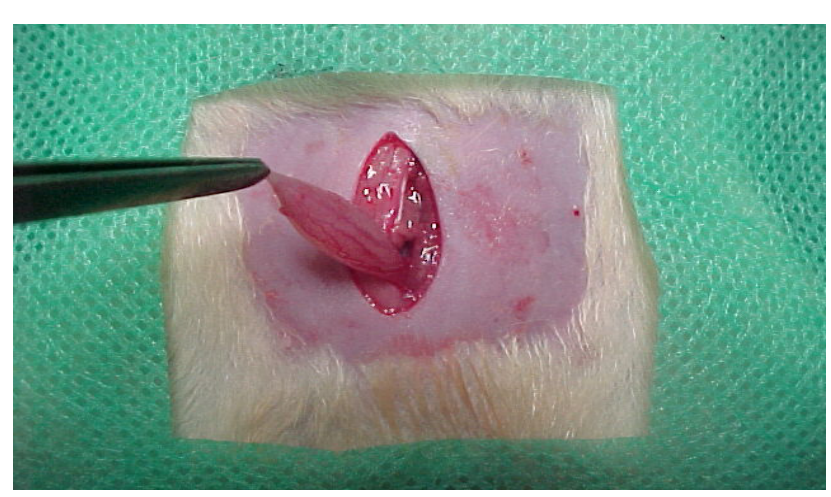

FIGURA 1 - Incisão mediana suprapúbica com exposição da bexiga

No pós-operatório, os animais eram mantidos com ração balanceada e livre acesso a água. Foram avaliados diariamente e anotadas as condições da ferida cirúrgica e possíveis complicações. O sacrifício foi realizado colocando-se cada animal em campânula de plástico com algodão embebido em éter, em sistema fechado, até que ocorresse o 
óbito. Foram sacrificados dez animais do GC e 10 animais do GA no $3^{\circ}$ dia pós-operatório, realizando-se o mesmo procedimento no $7^{\circ}$ dia.

\section{Avaliação macroscópica}

\section{Avaliação da parede e cavidade abdominal}

Iniciou-se com inspeção da cicatriz abdominal onde avaliou-se a presença de infecção e a integridade da sutura. Realizou-se em seguida abertura da cavidade abdominal através de três incisões. Duas transversais (uma suprapúbica e outra abaixo do apêndice xifóide) unidas por uma terceira lateral a incisão cirúrgica prévia, formando um retalho. Desta maneira obteve-se o acesso a cavidade abdominal, onde verificou-se a presença de sinais de infecção, aderências e o local da cistorrafia. Após tais observações procedeu-se a secção do colo da bexiga e sua retirada.

Para avaliação do grau das aderências utilizou-se o Escore de Nair ${ }^{9}$, conforme a Figura 2.

\begin{tabular}{|c|c|}
\hline Escore (Grau) & Achado \\
\hline $\mathbf{0}$ & Sem adesão \\
\hline $\mathbf{I}$ & $\begin{array}{l}\text { Adesão única entre dois órgãos ou entre um órgão e a parede } \\
\text { abdominal }\end{array}$ \\
\hline II & Duas adesões entre órgãos ou um órgão e a parede abdominal \\
\hline III & $\begin{array}{l}\text { Mais de duas adesões entre órgãos ou uma massa de adesão } \\
\text { generalizada do intestino sem aderir a parede abdominal }\end{array}$ \\
\hline IV & $\begin{array}{l}\text { Aderências generalizadas entre órgãos e a parede ou aderências } \\
\text { maciça entre todos os órgãos }\end{array}$ \\
\hline
\end{tabular}

FIGURA 2 - Escore de adesão de Nair

\section{Avaliação microscópica}

Procedeu-se a retirada de três fragmentos da bexiga, sendo dois paralelos a linha de sutura e um terceiro envolvendo-a. As amostras foram processadas pelas técnicas de coloração Hematoxilina e eosina (H.E.) e tricrômio de Masson.

Os critérios utilizados foram inflamação aguda, inflamação crônica, necrose isquêmica, reação gigantocelular tipo corpo estranho, reepitelização, coaptação das bordas da sutura, extensão do infiltrado da parede e neoformação capilar. Foram acrescentados os parâmetros proliferação fibroblástica e fibrose, também analisados com tricrômio de Masson.

\section{Análise estatística}

Para análise dos resultados utilizou-se o programa Statistica for Windows 5.1. As variáveis de avaliação microscópica foram analisadas pelo teste não-paramétrico de Mann-Whitney. O nível de significância utilizado para rejeitar-se a hipótese de nulidade foi a de 0,05 .

\section{Resultados}

\section{Avaliação macroscópica}

\section{Exame da parede e cavidade abdominal}

Foi observada infecção na ferida cirúrgica da parede abdominal em dois animais do SGC3, 17 do SGC7 e um do SGA3. Ambos os grupos apresentaram aderências com a bexiga, principalmente com o omento e parede abdominal (Tabela 1), havendo diferença estatisticamente significante ( $\mathrm{p}=0,013$ ) com maior freqüência no GA Aroeira que no GC no $7^{\circ}$ dia.

TABELA 1 - Grau de aderência segundo Escore de Nair

\begin{tabular}{ccccc}
\hline Escore de Nair & SGC3 & SGA3 & SGC7 & SGA7 \\
\hline $\mathbf{0}$ & 3 & 1 & 8 & - \\
$\mathbf{1}$ & 7 & 8 & 1 & 8 \\
$\mathbf{2}$ & - & 1 & 1 & - \\
$\mathbf{3}$ & - & - & - & - \\
$\mathbf{4}$ & - & - & - & - \\
\hline Total & 10 & 10 & 10 & 10 \\
\hline
\end{tabular}

Ao exame da bexiga não observaram-se abscessos, deiscências ou fistulas. As condições da sutura no $3^{\circ}$ e $7^{\circ}$ sétimo dias foram consideradas boas nos dois grupos.

\section{Avaliação microscópica}

\section{Terceiro dia de pós-operatório}

A Tabela 2 apresenta os resultados dos parâmetros histológicos do SGC e SGA no $3^{\circ}$ dia do pós-operatório.

TABELA 2 - Análise estatística dos parâmetros histológicos $-3^{\circ}$ dia

\begin{tabular}{lccc}
\hline Variáveis histológicas & SGC3 & SGA3 & p \\
\hline Inflamação aguda & 131.5 & 78.5 & $0.045^{*}$ \\
Inflamação crônica & 95 & 115 & 0.450 \\
Necrose & 119 & 91 & 0.290 \\
Gigantocelular & 108 & 102 & 0.821 \\
Fibroblastos & 83 & 127 & 0.096 \\
Colagenização & 59 & 151 & $0.001^{*}$ \\
Reepitelizaçao & 100 & 110 & 0.705 \\
Coaptação & 100 & 110 & 0.705 \\
Extensão & 105 & 105 & 1.000 \\
Neoformação & 55 & 155 & $0.000^{*}$ \\
\hline
\end{tabular}

No $3^{\circ}$ dia de pós-operatório, a reação inflamatória aguda foi moderada em três animais e acentuada em sete do SGC (Figura 3), enquanto que no SGA foi discreta em um animal, moderada em sete e acentuada em dois. Houve diferença estatisticamente significante entre os dois subgrupos.

A ausencia de colágeno ocorreu em nove animais do SGC e discreta em um, enquanto que no SGA foi discreta em oito e moderada em dois havendo diferença estatisticamente significante entre os dois subgrupos. 


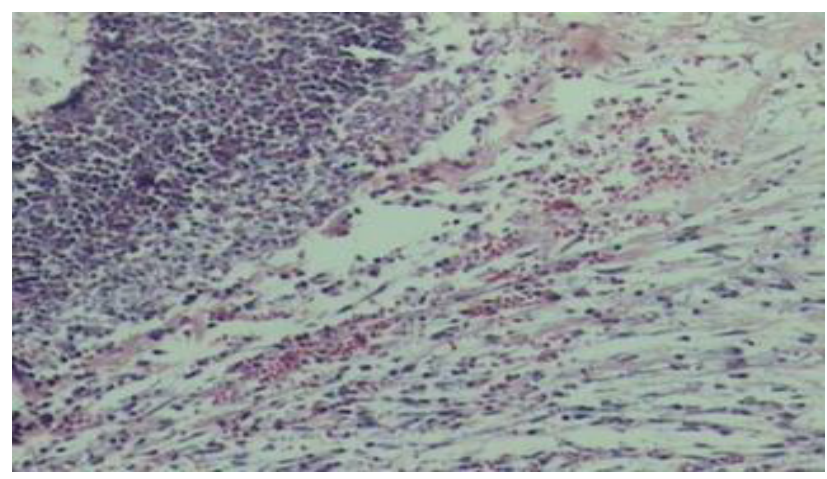

FIGURA 3 - Fotomicrografia de inflamação aguda. Legenda: $3^{\circ}$ dia pós-operatório SGC (coloração H.E., aumento 100x). Nota: 1= infiltrado de neutrófilos. 2 = área de edema

A neoformaçao vascular foi ausente em todos os animais do SGC, leve em nove animais e moderada em um do SGA, havendo diferença significativa entre os dois subgrupos.

Os demais parâmetros avaliados no $3^{\circ}$ dia, não apresentaram diferenças significativas entre os subgrupos analisados.

A colagenização foi ausente em nove animais do SGC e discreta em um, enquanto que no SGA foi discreta em oito e moderada em dois havendo diferença estatisticamente significante entre os dois subgrupos.

A neoformaçao vascular foi ausente em todos os animais do SGC, leve em nove animais e moderada em um do SGA, havendo diferença significativa entre os dois subgrupos.

Os demais parâmetros avaliados no $3^{\circ}$ dia, não apresentaram diferenças significativas entre os subgrupos analisados.

\section{Sétimo dia do pós-operatório}

A Tabela 3 apresenta os resultados dos parâmetros histológicos dos subgrupos SGC e SGA no $7^{\circ}$ dia do pósoperatório

TABELA3 - Teste não-paramétrico de Mann-Withney - $7^{\circ}$ dia do pós-operatório

\begin{tabular}{l|ccc}
\hline Variáveis histológicas & SGC7 & SGA7 & p \\
\hline Inflamação aguda & 130 & 41 & $0.004^{*}$ \\
Inflamação crônica & 64 & 107 & $0.006^{*}$ \\
Necrose & 80 & 91 & 0.183 \\
Gigantocelular & 85.5 & 85.5 & 0.399 \\
Fibroblastos & 106 & 65 & 0.328 \\
Colagenização & 94 & 77 & 0.929 \\
Reepitelização & 107 & 64 & 0.286 \\
Coaptação & 115 & 56 & 0.076 \\
Extensão & 95 & 76 & 1.000 \\
Neoformação & 59 & 112 & $0.001^{*}$ \\
\hline
\end{tabular}

A inflamação aguda foi moderada em todos os animais do SGC e ausente em um, discreta em seis e moderada em um do SGA. Houve diferença estatisticamente significante entre os dois subgrupos $(p=0,004)$ favorecendo o SGA.

A inflamação crônica foi discreta em nove animais e moderada em um do SGC enquanto que no SGA foi discreta em um e moderada em sete animais (Figura 4), havendo diferença estatisticamente significante $(\mathrm{p}=0,006)$.

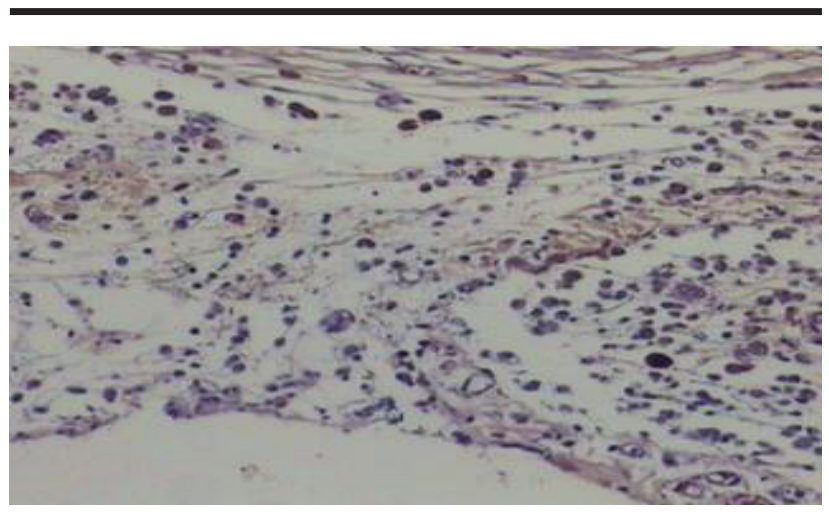

FIGURA 4 - Fotomicrografia do processo inflamatório crônico. Legenda: $7^{\circ}$ dia do pós-operatório, SGA(coloração HE., aumento 100x)

A neoformação vascular foi leve em nove animais e moderada em um do SGC e moderada em todos animais do SGA. Houve diferença estatística significante entre os dois subgrupos $(\mathrm{p}=0,001)$ favorecendo o SGA.

Nos demais parâmetros do $7^{\circ}$ dia analisados não foram observadas diferenças estatisticamente significantes.

\section{Discussão}

A incisão cirúrgica mediana longitudinal suprapúbica mostrou-se adequada para identificação e exposição da bexiga, como já relatado nos trabalhos de Holmes et al. ${ }^{10} \mathrm{e}$ Schauffert et al. ${ }^{11}$. Contudo, outra incisão é descrita por Ortiz et al. ${ }^{8}$ que utilizaram a transversa suprapúbica para exteriorização da bexiga de ratos também com bons resultados. A cistotomia de $1 \mathrm{~cm}$ foi suficiente para boa visualização das bordas e realização da cistorrafia. Foi utilizado fio absorvível sintético de poliglatina 5-0 para sutura da bexiga, em plano único, por ser adequado nos procedimentos cirúrgicos do trato urinário, devido às suas características de pouca reação inflamatória, evitando-se assim a formação de litíase ${ }^{13}$.

Houve infecção na parede abdominal em quatro animais. A infecção da ferida cirúrgica é a complicação pósoperatória mais comum e possui etiologia multifatorial, dependendo, dentre outros fatores, do tipo de operação, da duração da mesma e do estado imunológico do organismo do animal submetido ao ato operatório9.

Foram observadas aderências a semelhança dos estudos de Schauffert et al. ${ }^{11}$. Essa formação é considerada ocorrência inevitável, resultante do trauma na superfície peritoneal dos órgãos intraabdominais. O trauma inicia reação inflamatória, seguida por aumento da permeabilidade vascular, liberando exudato rico em fibrina. Se a fibrinólise não é efetiva, resulta na formação de aderência ${ }^{9,10,11}$. 
O exame histológico habitualmente utilizado para avaliação da cicatrização é baseado em dois métodos de coloração: o primeiro, hematoxilina-eosina, que permite identificar os tipos celulares envolvidos no processo inflamatório (mononuclear e polimorfonuclear), edema intersticial, congestão vascular, tecido de granulação e fibrose, e o segundo, tricrômio de Masson, indicado para identificação de fibras colagenas ${ }^{13,14,15}$. Para estudar as etapas da cicatrização escolheu-se o $3^{\circ}$ dia por representar fase inicial, caracterizada por processo inflamatório e o $7^{\circ}$ para avaliar os diversos parâmetros da fase intermediária da cicatrização $0^{9,16,17}$.

Pela análise histológica no $3^{\circ}$ dia a reação inflamatória aguda foi maior no SGC, enquanto que a colagenização foi mais acentuada no SGA. A neoformação vascular foi evidenciada apenas no SGA. No $7^{\circ}$ dia a inflamação crônica e a neoformação vascular foram mais intensas no SGA e o parâmetro inflamação aguda novamente mostrou-se mais acentuado no SGC. O grau de intensidade da reação inflamatória é de fundamental importância na cicatrização $0^{3,18}$. Assim, certo grau de inflamação é necessário, contudo uma reação infamatória intensa é prejudicial, pois pode haver comprometimento da microcirculação e ainda inibir a proliferação celular (fibroblastos). Camargo et al. ${ }^{19}$ constataram que o extrato alcoólico de guacatonga prolongou a fase aguda do processo inflamatório e conseqüentemente retardou os eventos típicos da fase regenerativa, ao contrário do observado com extrato hidroalcoólico da Aroeira neste estudo. Ficou evidenciado que alguns eventos presentes no processo de cicatrização foram favorecidos nos animais do SGA, comparativamente com o $\mathrm{SGC}^{20}$.

\section{Conclusão}

O uso de extrato hidroalcoólico de Aroeira mostrou efeito cicatrizante favorável nas cistotomias em ratos.

\section{Referências}

1. Brito MVH, Brito NMB, Cruz MMS, Oliveira RVB, Silva, TB, Reis JMC. Estudo Histológico da bexiga de ratos após administração de óleo de copaíba. Rev Par Med 1999;20-4.

2. Santos LC, Amorim MMR. Uso da Aroeira (Schinus terebinthifolius Raddi) para Tratamento de infecções vaginais. Femina 2002: 1-4.

3. Guerra, MJM. Barreiro ML, Rodriguez ZM, Rubalcaba Y. Actividad Antimicrobiana de um Extracto Fluido al $80 \%$ de Shinus Terebinthifolius Raddi (COPAL). Rev Cub Plant Med. 2000; 5:23-5.

4. Lisboa Neto JÁ, Machado J, Melo Jr. EJM, Raposa MJ. Avaliação do efeito cicatrizante da Aroeira (Schinus terebinhthfolius) e do mastruço (Chenopodium ambrosioides) em feridas de extração dental em ratos. Estudo Histológico. Rev Abo Nac 1998; :173-5.
5. Oliveira ST et al Formulações de confrei (symphytum officinale L.) na cicatrização de feridas cutâneas em ratos. Rev Fac Zootec Vet Agro, 2000; 7:61-5.

6. Amorim MMR, Santos LC. Tratamento da vaginose bacteriana com gel vaginal de Aroeira (Schinus terebinthifolius Raddi): ensaio clínico randomizado. RBGO 2003;25:95-102.

7. Jain MK, YU B, Rogers JM, Smith AE, Boger ETA, Ostrander RL, Rheingold, AL. Specific Comt]petitive inhibitor of Secretes Phospjolipase A2 from Berries of Shinus terebinthifolius. Phytochemistry 1995;537-47.

8. Ortiz V, Santos P, Osaki LT, Goldenberf S. Sutura de bexiga em plano único, extramucosos, empregando fio absorvível. J Bras Urol. 1986,131-3.

9. CorsiRC, Corsi RP, Pirana S, Muraco FAE, Jorge D. Cicatrização de feridas; revisão de literatura. Rev Bras Cir. 1994;84:17-24.

10. Holmes SAV. James M, Whitfield HN. Potential Use of Tissue Adhesive in Urinary Tract Surgery. Br J Utoly 1992; 647-50.

11. Schauffert MD, Simões MJ.; Juliano Y, Novo NF, Gomes PO, Ortiz V. Estudo da Ação dos fios Categute cromado e poliglecaprone 25 na ileocistoplasria em ratos, destacando a formação de cálculos. Acta Cir Bra s2000;237.

12. Costa MAR, Campos ACL, Coelho JCU, Barros AMB, Matsumoto HM. Oral Glutamine and the Heraling of Colonic Anastomoses in Rats. J Parenter Enteral Nutri. 2003:1-5.

13. Kuzu MA, Koskoy C, Kale T, Demiprence E, Renda N. Experimental study of the effect of preoperative 5fluorouracil on the integrity of colonic anastomoses. $\mathrm{Br}$ J Surg. 1998;85: 236-39.

14. Cohen IK, Diegelmann RF, Crossland MC. Os cuidados com a ferida. In: Sabiston Jr., D. C. Tratado de Cirurgia. Rio de Janeiro: Interamericana; 1986.

15. Benet LZ, Kroetz DL, Sheiner LB. "Pharmacokinetics: The dynamics of drug absorption, distribution, and elimination". In: Molinoff PB, Ruddon RW, editores. The pharmacological basis of therapeutics. McGraw-Hill; 1996.

16. Thornton FJ, Barbul A. Healing in the gastrointestinal tract. In: Barbul A. Wound healing. Surg Clin North Am. 1997; 77: 547-70.

17. Martinez MJ, Betancourt J,González NA. Actividad Antimicrobiana del Schinus terebenthifolius Raddi (COPAL). Rev Cub Plant Med 1996:37-9.

18. Camargo FG, Pereira, JÁ, BuenoVS, Gomes E, Ando T. Ação de Extrato alcoólico de guaçatonfa (casearia sylvestris sw) em subcutâneo de camundongo - Parte I - Estudo Histológico. LECTA/USF, 1995.

19. Ikeuchi D, Onodera H, Aung T, Kan S, Kawamoto H, Iamamura M, Maetani S. Correlation of tensile strength with bursting pressure in the evaluation of intestinal anastomoses. Dig Surg. 1999; 16: 478-85 


\section{Correspondência}

Periguari Luis Holanda de Lucena

Hospital São Domingos

AV. Jerônimo de Albuquerque, 540 - Bequimão - São Luís -MA

CEP: 65.060-642

Tel: (98) 3216-8510
Conflito de interesse: nenhum Fonte de financiamento: Capes

Recebimento: 22/01/2005

Revisão: 23/05/2005 Aprovação: 17/06/2006

\section{Como citar este artigo}

Lucena PLH, Ribas-Filho JM, Nascimento MM, Czeczko NG, Dietz UA, Correa-Neto MA, Henriques GS, Santos OJ, Ceschin AP, Thiele ES. Avaliação da ação da Aroeira (Schinus terebinthifolius Raddi) na cicatrização de feridas cirúrgicas em bexiga de ratos.Acta Cir Bras. [periódico na Internet] 2006; Suppl 2: 46-51. Disponível em URL: http://www.scielo. $\underline{\mathrm{br} / \mathrm{acb}}$

Figuras coloridas disponíveis em $\underline{\text { http://www.scielo.br/acb }}$ 